\title{
Le siècle des totalitarismes : éléments d'histoire conceptuelle et polémique
}

Marc Angenot ${ }^{1}$

\begin{abstract}
This article aims to reflect on multiple senses of the concept of totalitarianism inscribed in an historical and semantics approach that starts during the years 1920 through the emergence of the neologism in the Italian magazine "Il Mondo," and analyzing the ideological effects of totalitarian regimes according to their functions in different historical contexts: the Germany of Hitler, the Italy in Mussolini's regime and Russia in Stalin's regime. Considering the reflections on derivations and transformations that this concept acquires, we established a dialogue with the ideas presented by Hannah Arendt in her book "On The Nature of Totalitarianism" (1990), searching for a comprehension of the conceptual history of the term.
\end{abstract}

Keywords: totalitarianism; fascism; Begriffsgeschichte.

Resumo: Este estudo reflete sobre o funcionamento polissêmico da concepção de totalitarismo a partir de uma abordagem histórico-semântica que inicia durante os anos 1920 com a neologização do termo pelo jornal "Il Mondo", analisando os efeitos das ideologias dos regimes totalitários em seus diferentes funcionamentos nos contextos históricos da Alemanha hitlerista, da Itália no regime de Mussolini e da Rússia durante o regime stalinista. A partir de reflexões em torno das derivações e transformações que o conceito apresenta, estabelecemos um diálogo com as idéias de Hannah Arendt propostas em "As Origens do Totalitarismo" (1989) buscando uma compreensão da história conceitual do totalitarismo.

Palavras-chave: totalitarismo; fascismo; semântica histórica.

\section{Introduction}

«Totalitarisme», pour les uns, est une catégorie fondamentale en vue de définir le 20e siècle, mais c'est un terme tendancieux et peu explicatif pour les autres, et un catégorème indéfectiblement instrumentalisé par la droite, odieux pour d'autres encore, qui y voient une sournoise assimilation des régimes bolchevik, fasciste et nazi. La notion demeure un objet d'âpres controverses et de dialogues de sourds, qui ne semblent pas près d'être

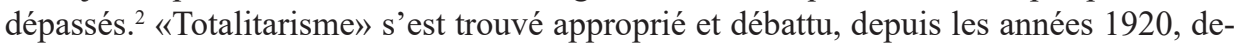
puis la prise de pouvoir des fascistes en Italie, par tous les courants politiques et toutes les écoles historiennes à leur suite - revendiqué ou dénoncé, défini et contre-défini, adopté comme susceptible d'éclairer le malheur des temps, mais tout aussi bien rejeté,

1 Professor Emérito da Universidade Mc.Gill, Canadá.

2 Simon Tormey, Making Sense of Tyranny: Interpretations of Totalitarianism. Manchester: Manchester UP, 1995. Voir aussi Totalitarismus und Faschismus. Eine wissenschaftliche und politische Begriffskontroverse. Kolloquium in Institut für Zeitgeschichte am 24 Nov. 1978. München: Oldenbourg, 1980. 
répudié, censuré. Le terme a été utilisé par tous les partis, tous les courants, du fascisme à l'antifascisme, du marxisme au libéralisme, de l'anarchisme au conservatisme, chacun lui attribuant une signification différente ${ }^{3}$. Depuis un bon demi-siècle, le catégorème a été régulièrement déclaré irrécupérable, dépassé, il a été mis de côté, et pourtant on l'a vu, à chaque fois, réapparaître, retravaillé et renouvelé. Après l'effondrement du Bloc de 1'Est et la «disparition» de 1'URSS en 1989-91, on a assisté à une résurgence tant soit peu triomphaliste des théories du totalitarisme qui étaient un peu tombées en déshérence. Les livres dans toutes les langues de l'Occident qui comportent dans leur titre même Totalitarianism, Totalitarismus, totalitarismo, totalitarisme se comptent par centaines. La catégorie a été plus d'une fois utilisée comme susceptible de résumer d'un seul mot, de ramasser en un mot le propre du 20e siècle, "das totalitäre Zeitalter», le siècle des totalitarismes. ${ }^{4}$ «Siècle des génocides», ajoutent d'autres ${ }^{5}$, «Siècle des camps»...

\section{L'histoire de la notion}

Dans un livre resté inédit, paru pour quelques collègues et amis en deux cahiers de recherche ${ }^{7}$, je soumets la notion à une étude d'histoire conceptuelle. Reinhart Koselleck et ses élèves ont développé un programme de recherche qu'ils ont dénommé Begriffsgeschichte; on dit en français histoire conceptuelle ou sémantique historique. L'historien allemand, mort en 2006, met cette [1]démarche aux fondements de tout travail historiographique. L'étude des concepts et de leur histoire est, pose-t-il, la condition minimale de la connaissance historique, tout autant que la définition de l'histoire comme étant liée aux sociétés humaines ${ }^{8}$.

Les querelles d'application, de définition, de pertinence, après 1945, ont toutes tourné autour des deux expressions, décrites comme accomplies dans leur inhumanité, de la catégorie : l'Union soviétique de Staline (pour d'autres, l'URSS tout au long de son histoire depuis 1917) et le Troisième Reich hitlérien qui venait d'être vaincu et anéanti. Les autres régimes désignés occasionnellement comme «totalitaires», à commencer par l'Italie fasciste, ont été certes discutés par les écoles historiennes, inclus pour les uns, rejetés à la marge pour les autres ou simplement passés sous silence.

3 Enzo Traverso, dir. Le totalitarisme. Le 20e siècle en débat. Paris: Seuil, 2001.

4 Titre de chapitre de Hans Maier, Das Doppelgesicht des Religiösen. Religion. Gewalt. Politik. Freiburg iB: Herder, 2004.

5 Bernard Bruneteau, Le siècle des génocides. Paris: Colin, 2004.

6 Alain Brossat, L'épreuve du désastre: le 20e siècle et les camps. Paris: Albin Michel, 1996.

7 Fascisme, totalitarisme, religion séculière : trois concepts pour le XXe siècle. Notes pour un séminaire d'histoire conceptuelle.. Le volume III Totalitarisme = \# 39 et \# 39bis, est divisé en 2 cahiers de 347 \& 245 pp. respectivement; ils sont sortis au cours de l'hiver 2015-16.

8 L'expérience de l'histoire. Paris: Gallimard/Le Seuil, 1997, 101. Voir une synthèse des méthodes et positions de l'école de Cambridge d'histoire intellectuelle, synthèse qui intègre et confronte les démarches de la Begriffsgeschichte allemande: Mark Bevir, The Logic of the History of Ideas. Cambridge: Cambridge UP, 1999. En domaine français, un des rares représentants d'une telle histoire Jacques Guilhaumou, historien d'Aix-en-Provence qui a surtout publié sur la Révolution française. Il a théorisé sa démarche dans Discours et événement : l'histoire langagière des concepts. Besançon: PU de Franche-Comté, 2006. Je me rapporterai aussi - parmi de nombreux autres travaux de synthèse qu'on rencontre surtout en anglais et en allemand - à un autre ensemble de textes définitionnels rassemblés cette fois par Enzo Traverso, Le totalitarisme: le 20e siècle en débat, publié au Seuil en 2001. Eckard Jesse a procuré encore à la fin du siècle passé un premier bilan dans Totalitarismus im 20. Jahrhundert. 
«Totalitarisme», «totalitaire» se trouve par ailleurs appliqué non pas seulement à des régimes établis et à des partis, à des «mouvements» qui les prépareraient, mais à des idéologies, des projets, des manières de penser même qu'on a pu désigner comme relevant de l'«esprit totalitaire», de la «pensée totalitaire». Toutes les idéologies totales du siècle passé, qu'elles soient classées de gauche ou de droite, ont créé des «vies inutiles», elles ont légitimé le meurtre de misérables par milliers, elles ont conçu et justifié le recours à la terreur en vue de soumettre la société à un remodelage intégral. C'est ici que l'on rencontre la question-clé qui est au cœur de mes recherches : celle du rôle des idées dans l'histoire.

Je vais me borner à rappeler quelques moments de la longue «Querelle du totalitarisme». Je m'attarderai aux étapes de l'émergence de la catégorie. Je veux montrer que l'histoire controversée d'une catégorie historiographique apprend beaucoup sur sa portée et son intérêt actuels. Je m'arrêterai en 1952 au «classique» de Hannah Arendt, mais il va de soi qu'il y aurait lieu de poursuivre jusqu'à aujourd'hui.

\section{Italie, années 1920, émergence de deux mots}

J'en viens donc au contexte d'émergence de deux mots attestés d'abord en italien, adjectif et substantif dérivé: totalitario, totalitarismo. «Totalitarisme» est en effet un mot-concept dont l'histoire commence exactement à la même époque, les années 1920, que «fascista, fascismo» et dans son environnement sémantique immédiat. Cette histoire commence dès lors bien avant la Guerre froide qui va instrumentaliser la dénonciation du «totalitarisme» au service de la lutte du «Monde libre» contre l'ex-allié stalinien. L'étude de la genèse entre les deux guerres de «totalitaire / totalitarisme» et de la relativement large et aussitôt controversée diffusion de ces mots invite à écarter la qualification fallacieuse de «concept de Guerre froide» qui a été imposée au terme par la sophistique anti-fasciste.

L'adjectif «totalitario» est néologisé en 1923 dans le journal Il Mondo par le libéral Giovanni Amendola en vue de dénoncer le régime qui se met en place en Italie et de stigmatiser les élections truquées de 1923. Amendola y definit le «sistema totalitario» inventé par les fascistes comme «promessa del dominio assoluto e dello spadroneggiamento completo ed incontrollato nel campo della vita politica ed amministrativa». Les fascistes qui viennent à peine d'accéder au pouvoir ont pour but ultime de s'approprier la conscience de chaque Italien. C'est l'accusation complémentaire de «religion politique» qui émerge : le fascisme prétend «être une religion» qui réclame la «conversion» de tous les Italiens.

Pour le dérivé «totalitarismo», la première attestation est datée de 1925. «Il totalitarismo fascista ha posto tutti i suoi principi: soppressione di ogni contrasto per il bene superiore della Nazione identificata con lo Stato, il quale si identifica a sua volta con gli uomini che detengono il potere» : ceci dans un article signé de Prometeo Filodemo (pseudonyme du socialiste Lelio Basso) dans La Rivoluzione liberale, 2 janvier 1925. Pour les fascistes-totalitaires, le bien de la nation s'identifie à la toute-puissance de l'État et de ceux qui le dirigent. C'est le noyau définitionnel stable jusqu'à la guerre à partir duquel la catégorie va s'étendre au delà du «cas» italien.

Néologisé par le libéral Amendola, le mot et son dérivé sont repris par les autres familles politiques exilées pour étiqueter et dénoncer le nouveau régime, pour caractériser, avec une certaine unanimité, «l'assujettisement inconditionné aux exigences du régime de toute forme de vie individuelle et collective», comme le définira un peu plus tard un 
autre libéral en exil, Silvio Trentin en faisant le bilan à Paris de «Dix ans de fascisme totalitaire» ${ }^{9}$. On voit que l'adjectif sert dans le titre même du livre à qualifier et définir le régime haï. Les catholiques émigrés car opposés au fascisme (non ceux, les plus nombreux, qui se sont accommodés du régime) n'ont pas manqué de s'en servir à leur tour. En 1924, le leader des Popolari, des démocrates chrétiens intransigeants, bannis par les fascistes, Don Luigi Sturzo, en exil depuis l'avènement de Mussolini, dénonce non sans perspicacité et prescience la «nuova concezione di stato-partito», l'idée de l'État-parti (autre néologisme en mot composé), entraînant une «trasformazione totalitaria di ogni e qualsiasi forza morale, culturale, politica e religiosa ${ }^{10}$. Pour le théologien qu'est Luigi Sturzo, le fascisme est une forme d'apostasie car il sacralise l'État et met la personne humaine au service de «faux dieux» temporels. Sturzo caractérise cette doctrine étatiste des fascistes comme étant «fondamentalement païenne et opposée au catholicisme : il s'agit de «statolâtrie» et de «déification de la nation», - l'expression sera reprise par le pape Pie XI dans l'encyclique Non abbiamo bisogno de 1931.

Ce qui va déterminer en l'infléchissant la diffusion première dans toutes les langues d'Europe de ces mots, c'est que Benito Mussolini, nullement embarrassé, au contraire, s'empare du néologisme réprobateur de ses adversaires en le revendiquant hautement. Il va exalter dans le discours fameux dit de l'Augusteo le 24 juin 1925 sa féroce volonté totalitaire, «la mia feroce volontà totalitaria». Et la définition du mot avancée par Mussolini n'est pas mauvaise du tout: rien d'humain ni de privé ne doit exister en dehors de l'État; l'État fasciste peut et doit englober et contrôler toute la vie sociale et spirituelle de la nation: totalitario résume bien l'axiome de la courte doctrine fasciste. Le régime de Mussolini - même si pour nous son totalitarisme pâlit face au nazisme et au stalinisme - est dès lors le seul qui se soit hautement déclaré totalitaire - et qui ait aimablement décoré du qualificatif des régimes amis, l'Autriche de Dollfuß, la Grèce de Metaxas et même l'URSS parfois ... quand il voulait d'aventure se montrer aimable envers les Bolcheviks. Le philosophe officiel du régime, Giovanni Gentile va s'offrir pour étendre un noble vernis hégélien sur le concept de «Stato totalitario». Gentile donne un tour philosophique à la définition de totalitarisme dans l'article «Doctrine du fascisme» qu'il écrit à la demande de Mussolini pour l'Enciclopedia Italiana. Il y expose que «pour le fasciste tout est dans l'État et rien d'humain et de spirituel n'existe et a encore moins de valeur hors de l'État. — En ce sens, conclut- il, le fascisme est totalitaire». ${ }^{11} \mathrm{Il}$ est aussi le premier intellectuel fasciste à qualifier philosophiquement, de façon approbative, le fascisme de «religion» politique.

Les opposants émigrés ont continué de leur côté à recourir à ces mots pour condamner le régime. Don Sturzo conclut qu'on ne pouvait désormais plus avoir aucun espoir «que le rythme totalitaire vienne à varier, que le rêve dictatorial s'effondre, que notre régime constitutionnel et notre base démocratique reviennent».11 ${ }^{12}$ Le fascisme et le christianisme sont inconciliables et Don Sturzo se refuse à croire que les avances faites par Mussolini allaient amollir ou faire vaciller le Vatican, l'Église ne se concilierait jamais avec un tel régime! Il allait devoir déchanter.

9 Dix ans de fascisme totalitaire. Paris, Éd. sociales internationales, 1937, p. 9.

10 Luigi Sturzo, «Spirito e realtà», 22 janv. 1924.

11 Giovanni Gentile, «Fascismo (dottrina del)», Enciclopedia Italiana, Istituto dell'Enciclopedia Italiana, Roma, 1932, vol. XIV, 835-840.

12 Cité par Emilio Gentile, Pour ou contre César? Les religions chrétiennes face aux totalitarisme. Paris: Aubier, 2013. 146. 
Une autre idée qui a un long avenir s'esquisse dans les écrits du prêtre-politicien. Le rapprochement entre le régime du Duce et l'URSS, dictatoriale et athée, s'impose à son esprit, ce qui l'invite à étendre le concept de «totalitarisme». Les deux régimes, rouge et noir, apparemment en conflit, sont semblables sur l'essentiel: «Il n'y a qu'une différence vraiment marquante, à savoir que le bolchevisme ou dictature communiste est le fascisme de gauche, tandis que le fascisme ou dictature conservatrice est le bolchevisme de droite» ${ }^{13}$. Sturzo met le premier en parallèle le caractère «totalitaire» de l'Italie mussolinienne et de la Russie bolchevique. Ce parallèle deviendra le lieu commun des publicistes catholiques jusqu'à la guerre. Or, quand Mussolini se réclame en 1925 d'un projet totalitario pour répudier l'État «libéral» faible et inefficace qu'il a abattu, l'URSS n'a pas encore atteint le stade que les historiens futurs qualifieront de «totalitaire» - quoique le parti unique et la terreur comme moyen de gouvernement sont déjà bien installés dans la Russie léniniste.

\section{Les années trente - Les catholiques face aux totalitarismes rouge, noir et brun}

L'émergence de la théorie du totalitarisme s'est faite dans le cadre d'une lecture prédominante, à deux dimensions, des combats de l'époque : «fascisme/antifascisme»,avatar bolchévisé du paradigme fondateur de la gauche: contre-révolution/révolution. Tout rapprochement entre le ou les fascismes et le régime soviétique, toute analyse de la nature «totalitaire» de l'URSS sont alors violemment dénoncés dans ce cadre binaire dans lequel «il n'y a pas de milieu» puisque la «démocratie formelle bourgeoise» «n'est qu'un leurre» proviosire. Du fait de la «vigilance» du Front antifasciste, quiconque compare et rapproche les deux régimes est rejeté dans les ténèbres extérieurs. L'antifascisme, la dénonciation concomitante de l'anti-communisme seront - et demeureront longtemps après la guerre - un moyen efficace d'intimidation et de censure des social-démocrates et des libéraux comme ils sont un moyen de diffamation et diabolisation des courants «oppositionnels», spécialement des infâmes «hitléro-trotskystes». Depuis 1935 et jusqu'au milieu des années 1970 (en dépit du Pacte nazi-soviétique 1939-1941), l’URSS doit paraître aux yeux des progressistes comme le paladin de l'antifascisme, et plus que jamais quand elle apparaîtra en outre après 1945 comme le principal vainqueur du nazisme et ce, au prix d'immenses souffrances. Le système communiste de l'Est en tirera sa justification morale, par delà les échecs et les «excès» parfois concédés, justification qui persistera vaille que vaille jusqu'au jour de son dépôt de bilan. Le refoulement intimidateur de la catégorie du «totalitarisme», attribuée après 1950 à une poignée de Cold Warriors au service de l'impérialisme, était indispensable au maintien de ce positionnement.

«Totalitarisme», ce fut alors surtout entre les deux guerres le mot-clé des milieux catholiques, une catégorie qui leur permettait précisément de ne pas se laisser enfermer dans le paradigme fascisme/antifascisme tout en ne se laissant pas séduire non plus par la propagande de Berlin qui faisait du nazisme le «rempart contre le bolchevisme». Plusieurs catholiques cependant, notamment ceux qui se rallient au personnalisme chrétien de Mounier, ajoutaient que c'est l'État libéral et laïc qui, par son matérialisme et ses carences, avait engendré la réaction totalitaire.

C'est le philosophe Waldemar Gurian, juif russe converti au catholicisme, écrivant en allemand, qui le premier se met en devoir de théoriser de façon approfondie le «totalita-

13 Op. cit., 221. 
risme» en plaçant au centre de ce régime nouveau, inouï, un groupe d'idéologues pénétrés d'une foi absolue, la «religion totalitaire», et régnant par la terreur sur les masses, l'État tout puissant n'étant pas le but, mais le moyen de réaliser un projet eschatologique et utopique. Les totalitarismes dont le caractère religieux-séculier est dès lors essentiel ne conçoivent pas un secteur de la vie qui puisse échapper à leur contrôle ni ne tolèrent de doctrines concurrentes. En vue de développer la théorie, Waldemar Gurian élabore le composé - qui a également un long avenir jusqu'à nous - d' «État-Parti».

Ce qui le rend insupportable et inaudible à gauche, c'est que Gurian développe systématiquement le parallèle entre communisme et nazisme. Ce, avant même la prise de pouvoir du NSDAP, dans

Le bolchevisme, danger mondial. ${ }^{14}$ Son axiome, typique d'un esprit spiritualiste, est qu'une société qui a rejeté la transcendance ne peut que concocter des ersatz, des religions séculières qui aboutissent à l'instauration d'une tyrannie dogmatique, c'est à dire précisément un «totalitarisme». Gurian en 1933 se sert des penseurs anti-démocrates allemands pour caractériser l'URSS qui à son sentiment sert de modèle, à la fois haï et émulé, aux nazis: «la formule significative d'Ernst Jünger de "mobilisation totale" et la thèse de Carl Schmidt de virement vers l'État total qui ne connaît plus l'ancienne distinction de l'État et de la société sont depuis des années des réalités dans l'État bolchevique. L'État fasciste, contraste-t-il [l'Italie vient de signer le Concordat avec Pie XI] n'est pas, de loin, aussi "total" que le bolchevisme» ${ }^{15}$. Gurian prétend distinguer les dictatures totalitaires des régimes autoritaires et des tyrannies «classiques» comme ceux de Franco ou de Mussolini: ceux-ci, si blâmables et brutaux soient-ils, n'essayent à tout le moins pas de remplacer le christianisme par une religion de l'immanence. Bien que son œuvre s'emploie à établir des similitudes qu'il présente comme essentielles entre les deux régimes, il ne fait aucun doute que pour Gurian en 1935 le principal danger en Europe est d'abord hitlérien, ... mais les raisons qu'il avance sont «originales». Les nazis sont des sortes d'hyper-bolcheviks qui ont éliminé les restes d'humanisme et de rationalisme qui subsistent vaille que vaille dans l'idéologie adverse ! Les thèses du philosophe sur les similarités et même la convergence des totalitarismes rouge, noir et brun ont été bien assimilées par le monde catholique pour lequel Gurian fournissait une explication satisfaisante du malheur des temps tout en dissuadant le chrétien, ému par les souffrances de la classe ouvrière, sensible à l'injustice sociale et soucieux de lutter contre les horreurs du fascisme, de se rapprocher des communistes.

En dehors des milieux catholiques, de tels essais qui tracent ce parallèle communisme/nazisme comme de semblables mouvements sanguinaires, anti-chrétiens et antihumanistes n'auront aucune influence. Ils ne seront simplement pas lus, écartés avec mépris et indignation là où s'imposait le paradigme antifasciste manichéen, celui de la lutte finale du Bien (même embarrassé de «contradictions» transitoires) contre le Mal.

Le personnalisme chrétien forme un courant d'idées français élaboré surtout par Emmanuel Mounier autour de la revue Esprit, lancée en 1932. Il prétend rechercher une troisième voie «humaniste» entre le brutal libéralisme capitaliste et le «totalitarisme»

14 Voir: Gurian, Der Bolschewismus: Einführung in Geschichte und Lehre. Freiburg iB: Herder,1931. > Bolshevism: An Introduction to Soviet Communism. Notre Dame IN: Notre Dame UP, 1952. > Le bolchevisme. Introduction historique et doctrinale. Paris: Beauchesne, 1933. + Bolschewismus als Weltgefahr. Luzern: Vita nova, 1935. > Le bolchevisme, danger mondial. Paris: Alsatia, 1933.

15 Le bolchevisme. Introduction historique et doctrinale, 3. 
sous ses deux formes antagonistes, marxisme et fascisme. «Totalitarisme» est alors utilisé dans les pages du journal chaque fois que ces régimes sont confrontés. Le personnalisme naît de deux ou plutôt trois refus, refus du libéralisme exploiteur, rupture avec ce qu'il nomme le «désordre établi», et refus tout d'un tenant du marxisme et du fascisme. Les personnalistes qui se disent hostiles à l'exploitation capitaliste et en montrent les horreurs, refusent complémentairement les tentatives «étatistes» du communisme ou du fascisme parce qu'elles broient l'individu et nient la «primauté» de la personne. La revue Esprit est toutefois isolée dans l'avant-guerre: la gauche laïque et réformatrice ne pense pas que toutes ses analyses sont fausses, elle peut avouer bien des «réticences» face à l'URSS, mais elle dénonce dans son triple refus qui implique ipso facto le rejet de toute coalition «antifasciste», de toute entente avec les communistes, un aveu d'impuissance au milieu de ce qu'on appelle la «montée des périls».

À la fin des années 1930, Rudolf Hilferding, qui était, lui, un des théoriciens connus de la social- démocratie allemande, en exil en France, relance à son tour l'expression d' «État totalitaire» pour dénoncer l'État NSDAP mais aussi pour caractériser le régime soviétique qui n'est selon lui ni un État socialiste, ni un capitalisme d'État, «mais un État où la politique commande à tout, à l'économie comme à la société.» ${ }^{16}$

Y avait-il un dénominateur commun à tous ces emplois du même mot par des observateurs très divers dans les années d'entre-deux guerres ? Il me semble que oui. «Totalitarisme», extrapolé selon les cas de l'observation de l'Italie fasciste, de la Russie bolchevique et après 1933 et même avant la prise de pouvoir du NSDAP, de l'Allemagne nazie, et de leur confrontation, désigne à tout coup un régime où l'État est censé absorber ou aspire à absorber la société civile, où il cherche à prendre le contrôle non seulement de l'économie, mais des institutions de toutes natures et à se soumettre les esprits de tous, un État aux mains d'une minorité prête à tout pour s'assurer d'un contrôle aussi étendu que possible. Cette minorité est mue par une idéologie «totale», omnisciente et indiscutable, qui se trouve qualifiée de diverses manières - notamment religieuse - où censément elle puise son droit irrévocable à occuper le pouvoir et exercer ce contrôle qui ne recule devant aucun moyen. À ces égards, le «totalitarisme» apparaît non comme un régime stabilisé, mais comme un processus en cours, qui n'a ni fin ni cesse, où les observateurs entrevoient une dynamique inexplicable et inextinguible.

Les auteurs de l'entre-deux-guerres n'opposent pas tous le «totalitarisme», bolchevik ou «fasciste», à la démocratie, à une démocratie idéalisée. Concurremment, ils l'inscrivent dans une hiérarchie des dictatures, des régimes autoritaires où il occuperait le degré suprême. La démocratie bourgeoise libérale léguée par le $19 \mathrm{e}$ siècle, là où elle était plus ou moins implantée, n'a guère résisté aux massacres de la guerre si ce n'est en Angleterre, en France, en Suisse et dans le Benelux. Dans les années 1930, sont établies neuf ou dix dictatures de droite dont deux seulement, l'italienne et la nazie, seront classées «fascistes» par tous les historiens. Pratiquement toute l'Europe centrale, orientale et méridionale est dotée de régimes à la fois autoritaires et réactionnaires, expressément contre-révolutionnaires. Aucun mouvement communiste ne renverse une démocratie «bourgeoise» entre 1918 et 1945 si ce n'est l'éphémère République des conseils de Béla Kun en Hongrie.

16 Alain Bergougnioux et Gérard Grunberg. L'utopie à l'épreuve, le socialisme européen au 20e siècle. Paris: De Fallois, 1996, p. 95. Hilferding est capturé à Arles en février 1941 par la police vichyste et livré à la Gestapo. Interné à la prison de la Santé, Hilferding s'est probablement suicidé dans sa cellule le 10 février 1941. 
Tous les régimes parlementaires renversés le sont par des coups de main qualifiés en leur temps de «fascistes» par la gauche - qui entretenait de la catégorie, il est vrai, une vision très large. La Crise économique de 1929 ne se déclenche qu'alors que des «fascistes» sont au pouvoir, ou tout près de l'être, dans plus de la moitié des pays européens.

\section{Application de «totalitarisme» à I'URSS par les trotskystes}

Le courant révolutionnaire qui va explicitement développer le rapprochement de l'URSS de Staline avec le fascisme italien puis avec le nazisme et en tirer les conséquences politiques est le trotskysme. Dès 1933, Victor Serge, qui venait de l'anarchisme et avait rejoint les trotskystes, voit dans l'URSS non une quelconque construction en marche du socialisme mais, ce sont ses mots, «un État totalitaire, "castocratique", absolu, grisé de puissance, pour lequel l'homme ne compte pas». Serge est ainsi le premier théoricien connu à l'extrême gauche à qualifier l'URSS d' «État totalitaire». ${ }^{17}$

J'aurais pu remonter aux inquiétudes exprimées parmi plus d'un leader de l'Internationale face à la prise de pouvoir des bolcheviks, à leur violence excessive immédiate, à certaines mesures répressives brutales prises dès 1918 en Russie ; $j$ 'aurais pu m'arrêter aux cris d'alarme de Karl Kautsky et à ceux Rosa Luxemburg en vue de dégager une généalogie des critiques du bolchevisme à l'extrême gauche - ce n'est pas mon objet ici.

L'idée et la qualification avancées par Victor Serge se trouvent reprises et illustrées dans Vers l'autre flamme, livre signé par Panaït Istrati, mais dont on sait qu'il a été partiellement rédigé par Victor Serge et Boris Souvarine,$^{18}$ livre qui est un récit de voyage en URSS et un réquisitoire informé contre le Grand mensonge soviétique. Victor Serge répétera sa thèse jusqu'à sa mort prématurée. Léon Trotsky lui-même se sert de «totalitarisme» dans son livre de 1936 La Révolution trahie en vue de qualifier le stalinisme et le nazisme de «phénomènes symétriques», d'adversaires apparents d'une «mortelle similarité».

De même encore, le terme est adopté par Boris Souvarine, exclu du Parti communiste en 1924, l'intellectuel anti-stalinien par excellence, qui en 1935 publie sa terrible biographie de Staline. Souvarine qui a recours à la catégorisation totalitaire depuis les années 1930 publie en 1947 une brochure de synthèse dans Spartacus, intitulée Socialisme ou totalitarisme: l'URSS est à son jugement devenue un État «totalitaire», ce qui revient à dire qu'elle est radicalement anti-socialiste et contre-révolutionnaire et qu'un véritable révolutionnaire doit la combattre sans hésiter. ${ }^{19}$

En 1939, Otto Ruhle, qui avait été proche de l'ultra-gauche «conseilliste» dirigée par Antoon Pannekoek, pousse plus loin la comparaison, jusqu'à en renverser les termes: c'est la Russie qui est «au premier rang des États totalitaires», le fascisme n'est que la

17 Extrait de Hommage à Victor Serge, Spartacus, série B №50, oct. nov. 1972. Victor Serge, de son vrai nom Viktor Lvovitch Kibaltchiche né à Bruxelles en 1890 de parents russes, mort dans la misère à Mexico en 1947, était un révolutionnaire de tendance anarchiste qui s'était rapproché de Trotsky.

18 P. Istrati, Vers l'autre flamme. Après seize mois dans l'U.R.S.S. Paris: Riëder, 1929. Réed. Paris: U.G.E., 1980 .

19 Brochure rééditée dans : Serge et Souvarine, Le Nouvel impérialisme russe : l'Europe au carrefour, renaissance ou totalitarisme; L'URSS est-elle un régime socialiste / Serge. Hommage à Victor Serge : pour le 25 e anniversaire de sa mort / René Lefeuvre, Max Schachtman, Magdeleine Paz, Lucien Laurat. Le Stalinisme : ignominie de Staline / Boris Souvarine. Paris : Spartacus, 1972. 
pâle copie réactionnaire du bolchevisme, et la lutte contre le fascisme doit commencer par la lutte contre le bolchevisme. La signature du pacte germano-soviétique en 1939 lui semblera mettre la preuve sur la somme: la convergence des deux régimes est «la base même de leur alliance». Autre figure non-orthodoxe de la gauche des années 1930, la catholique Simone Weil analyse elle aussi expressément l'URSS comme «système totalitaire». On observe donc bien le retour de ce même mot qui a été apparemment indispensable aux esprits critiques.

Les rapprochements, quant à la misère sexuelle des masses aliénées, entre URSS/ Allemagne nazie se rencontrent encore chez le freudo-marxiste Wilhelm Reich dans sa Die Massenpsychologie des Faschismus ${ }^{20}$ qui sera réédité et relu passionnément par la jeunesse de 1968. Et le rapprochement des idéologies de ces régimes comme peur névrotique de la liberté reviendra peu après chez Erich Fromm, dans Escape from Freedom. ${ }^{21}$

Il serait difficile alors de nier que «totalitarisme» a eu droit de cité dans toute la gauche radicale non soumise à l'orthodoxie staliniste - et le mot a favorisé le rapprochement, étayé et élaboré, entre stalinisme et régime nazi. D’autres trotskystes et oppositionnels, en vue de caractériser l'URSS stalinienne, qui n'était ni capitaliste, ni vraiment socialiste, emprunteront toutefois, plutôt que ce mot qui pouvait apparaître d'origine «bourgeoise», un concept marxien exhumé par Karl Wittfogel, celui de «despotisme asiatique» ou «despotisme agraire». À ces critiques s'opposent toutefois ceux, sans doute les plus nombreux, qui, comme le bon historien américain «de gauche» Hans Kohn en 1935, ont cherché obstinément, désespérément, à maintenir une distinction entre le nazisme, mal absolu, et le stalinisme, mal transitoire dans une URSS susceptible en dépit de tout de se réformer quelque jour et de reprendre la voie du socialisme.

Après l'entrée en guerre de l'URSS aux côtés des Alliés en 1941 et la victoire russe chèrement payée de 1945, la condamnation radicale de l'URSS et le rapprochement du régime de Staline avec le nazisme anéanti ne sera plus le fait que de rares oppositionnels, isolés et diffamés, suspects à toute la gauche ... et pas tellement appréciés de la droite. Ceci, avant que la comparaison ne retrouve sa place dans l'arsenal des politologues de la Guerre froide - et avant de faire retour en force à la fin des années 1970 et à titre de prélude à la dissolution du «Bloc de l'Est».

\section{L'«État total»chez les penseurs pré-nazis}

On assiste au cours de la dernière décennie de l'entre-deux-guerres à une translation géopolitique du champ lexical des dérivés de «total» et à leur réinterprétation, de l'Italie fasciste à l'Allemagne pré-nazie. Les syntagmes politiques dérivés de «total» ont en effet une complexe histoire d'emprunts et d'adaptations qu'on pourrait étendre à toute l'Europe antidémocratique de l'entre- deux-guerres. Ces formules ont été étudiées dans le livre pionnier de Jean-Pierre Faye, Langages totalitaires. ${ }^{22}$ Vers 1930 «total-/itaire», venu d'Italie, trouve un écho en Allemagne, davantage parmi des universitaires comme «le grand juriste» Carl Schmitt que chez les dirigeants du NSDAP - qui ne sont guère des têtes théoriques.

20 Kopenhagen, Prag, Zürich: Verlag für Sexualpolitik, 1933-1934. > La psychologie de masse du fascisme. Paris: Payot, 1972.

21 New York: Farrar \& Rinehart, 1941. > Rééd. sous le titre The Fear of Freedom. London: Routledge \& Kegan Paul, 1942.

221972. 
Paraît d'abord en 1930 un livre d'Ernst Jünger, écrivain devenu célèbre après la publication d'Orages d'acier, ses souvenirs de la Première Guerre mondiale. Cet essai de doctrine a pour titre: Der Weltstaat. Die totale Mobilmachung, L'Etat universel suivi de : La mobilisation totale ${ }^{23}$ Jünger y élabore le premier le concept de Totale Staat en développant concurremment l'idée, tirée de son expérience de la guerre, de Totale Mobilmachung, de mobilisation totale de la nation mais en temps de paix - le temps de paix n'étant jamais qu'une préparation à la guerre. Jünger part de la mobilisation de toute la population que la Guerre de 1914 a imposée à tous les pays européens et des «enseignements» qu'on peut tirer de cette dynamique inouïe transposable au transitoire temps de paix. Tous peuvent être et seront mobilisés. ${ }^{24}$ La guerre moderne est envisagée par Jünger comme un processus de travail, gigantesque dans ses dimensions. Il mobilise l'ensemble des réserves des peuples en guerre. Les pays se transforment en fabriques géantes qui produisent à la chaîne pour les armées. Un nouveau type d'homme est formé dans un tel contexte, celui du Travailleur-soldat, chez qui il ne reste rien de la poésie traditionnelle du soldat, héroïque et patriote, et qui ne jette plus son enthousiasme mais son professionnalisme acquis par un rigoureux entraînement dans la guerre totale. ${ }^{25}$ Homme de lettres figure majeure de la «révolution conservatrice» à l'époque de Weimar, Jünger s'est tenu éloigné de la vie politique à partir de l'accession des nazis au pouvoir - ce qui a permis à son «image» et à son œuvre de survivre vaille que vaille à la guerre.

La diffusion en Allemagne de locutions de prospective politique comportant «- total» est accentuée par le succès concomitant du livre du «glorieux» général Ludendorff, Der totale Krieg, La guerre totale (1935) qui subvertit la conception de Clausewitz de la guerre comme continuation de la politique par d'autres moyens, pour la transformer en une vision la paix comme préparation à la guerre et de «la politique comme continuation de la guerre par d'autres moyens». ${ }^{26}$

Le projet d'établir un «État total» apparaît donc chez tous les penseurs des années 1920-30 qu'on peut voir comme des fourriers du nazisme, dont au premier rang Carl Schmitt (1888-1985). Ce projet découle du diagnostic négatif préalable qu'ils font tous à l'égard de l'ordre libéral pluraliste avec son État faible, juridiquement limité et contraint face aux individus et aux intérêts conflictuels de la société civile, cet ordre légué par le 19e siècle bourgeois qui, après la Guerre mondiale, est jugé impuissant, sans force ni volonté, incapable de faire face aux défis de l'époque. La Guerre mondiale a brutalement démontré hors de tout doute les insurmontables faiblesses du parlementarisme, du régime de partis pour un peuple quand il se trouve mis face à des dangers vitaux. Son impuissance pratique et son désaveu moral à leur sentiment les invitent à spéculer sur l'instauration nécessaire et urgente d'un ordre tout contraire qui assurera l'Unité d'un peuple énergique et toujours mobilisé. Un ordre où société civile et État fusionneront dans une version autoritaire de la Volonté générale confondue avec la «force vitale» qui émane du Volk. C'est en quoi, ils se voient, complaisamment, répudiant l'ordre passé, comme des «révolutionnaires» à leur manière. Schmitt, à la fin de la République de Weimar, théorise ce qu'il qualifie d'«État qualitativement fort» ou «État total qualitatif», il appelle de ses vœux

23 L'Etat universel suivi de La mobilisation totale. Paris : Gallimard, 1990.

24 L'État universel..., 108-112.

25 Résumé et paraphrasé d'après Wolfgang Herrmann, Der neue Nationalismus und seine Literatur, San Casciano Verlag, Limburg, notice «Ernst Jünger».

26 Juan Linz, Régimes totalitaires et autoritaires. Préf. de Guy Hermet. Paris: Armand Colin, 2006, 20. 
cet État nouveau qui incarnera la Souveraineté, qui souverainement décidera de l'ami et de l'ennemi. Et pour Schmitt, cet État total allemand - l'État mussolinien qui a montré la voie après la guerre cesse de l'inspirer car regrettablement, il n'est pas raciste - État affranchi de toute entrave par le piteux «droit» libéral devra être un État racial, un völkische Staat.

Carl Schmitt adhère en 1933 au Parti nazi lequel est tout heureux d'avoir rallié à la Cause un juriste de notoriété internationale. La théorie de l'État total est inséparable du paradigme non moins fameux de Schmitt, qui réduit la politique à son état le plus archaïque: la distinction entre hostis et inimicus (ennemi politique vs ennemi privé), la relation axiomatique ami-ennemi formant la base de tout jugement et de toute décision politiques et étant rigoureusement indépendante des notions morales «privées» de bien et de mal. Schmitt s'inspire à la fois des écrits de son ami Ernst Jünger et de l'expérience italienne qu'il admire immensément, il voit dans le fascisme transalpin un régime antibourgeois analogue au bolchevisme mais qui répond avec vigueur à la menace que ledit bolchevisme fait peser, qui répond à la guerre civile internationalisée prônée par le Komintern par une «guerre civile d'État». ${ }^{27}$

Complémentairement, Schmitt élabore un autre concept qui deviendra fameux, celui de «säkularisierte Theologie», de théologie sécularisée. À la façon de Giovanni Gentile pour Mussolini, il procure aux dirigeants nazis qui en ont bien besoin, un cadre de haute philosophie qui leur permet de sacraliser l'idéologie du régime et de faire porter le soupçon sur le rationalisme des Lumières. Schmitt est en effet le penseur de la «théologie politique» dont on connaît le théorème qui demeure aujourd'hui enseigné partout: «Tous les concepts prégnants de la théorie moderne de l'État sont des concepts théologiques sécularisés».$^{28}$ Avec Schmitt, les choses sont claires: la thèse est une machine polémique contre la démocratie libérale et sa prétention à être fondée en raison séculière. Elle procure une mise en question radicale de la «légitimité des temps modernes»: les concepts centraux de la pensée politique moderne sont du théologique perpétué et dénié. La thèse prétend dénoncer une dérivation non assumée: le sentiment de rupture avec la pensée religieuse affiché par la modernité politique est une imposture.

Les théories de Carl Schmitt, qui se montre un admirateur, à sa façon nazie, de l'URSS stalinienne dans ce qu'elle a précisément de brutal et de «total», serviront à plus d'un essayiste anti-totalitaire de l'entre-deux-guerres pour leur confirmer la convergence, l'identité essentielle des conceptions bolcheviques et nazies: «De grands écrivains nationaux-socialistes, remarque Waldemar Gurian, ont eux-mêmes reconnu et formulé, avec une objectivité remarquable, la concordance entre l'État soviétique et le Troisième Reich. Carl Schmitt, le juriste qui fait loi en Allemagne, dans son ouvrage Staat, Bewegung, Volk (État, Mouvement, Peuple) fondamental pour le droit public national-socialiste, voit expressément dans l'Union soviétique l'État-type du 20e siècle, comme formellement opposé à l'État-type du 19e siècle. Le «mouvement», c'est-à-dire un parti qui soutient l'État et dirige en même temps le peuple, la classe dirigeante qui est à la fois soutien du gouvernement et représentant du peuple, constitue, à l'encontre de ce qui existait au $19 \mathrm{e}$ siècle, une unité strictement disciplinée, qui reste en un vivant contact avec le peuple, c'est-à-dire avec la masse des travailleurs. Certes le national-socialisme n'est pas un

27 Chaunu, Le paradigme totalitaire. Paris: F.-Xavier de Guibert, 2009. 16. Lire aussi David Cumin, Carl Schmitt, Biographie politique et intellectuelle, Paris: Cerf 2006.

28 Politische Theologie. Vier Kapitel zur Lehre von der Souveränität paraît en 1922. 
mouvement marxiste et la doctrine de Karl Marx n'en constitue pas la base, la chose est trop évidente, mais cela n'empêche nullement de le rapprocher du bolchevisme russe. N'y voit-on pas la guerre de domination et l'État-type, tel que le réalise le bolchevisme à base d'idéologie marxiste ? On ne peut donner une réponse valable à cette question que si l'on distingue d'une façon bien nette les différences entre les deux. Alors seulement il ressort avec évidence que les deux mouvements aboutissent à un même résultat, auquel le bolchevisme a donné son nom, parce que c'est dans l'Union soviétique qu'il s'est d'abord manifesté et a pris corps. $\gg{ }^{29}$ Carl Schmitt est le doctrinaire du totalitarisme en sa version nazie. Toutefois, la catégorie qu'il élabore est celle de Totale Staat - non un dérivé d'une langue romane comme eût été totalitär qui est aussi attesté ici et là. Le syntagme résout un problème de nationalisme lexical ! Le totale Staat hitlérien vient prendre la relève sous forme amendée du Stato totalitario fasciste. C'est que le mot totalitär n'est pas hitlérien: il est rejeté comme «trop libéral» par le juriste nazi Wilhelm Stuckart, dont Eichmann sera le subordonné. ${ }^{30}$

\section{Du Pacte Hitler-Staline à 1945}

Le 23 août 1939, Hitler et Staline concluent alliance. Le protocole secret du Pacte nazi-soviétique organise le partage de l'Europe. Pour Hitler, la Pologne occidentale et la Lituanie. Pour Staline, l'Estonie, la Lettonie, la Pologne orientale et la Bessarabie roumaine. Hitler se trouve libre d'attaquer la France et le Benelux. Puis ce seront la Yougoslavie et la Grèce. Staline est libre, lui, d'attaquer la Finlande. Sur les atrocités commises par l'Armée rouge en Pologne et dans les pays baltes à la faveur du pacte avec Hitler, on peut lire désormais les chapitres fondés sur archives de Roger Moorhouse, The Devil's Alliance. Hitler's Pact with Stalin 1939-1941. ${ }^{31}$

Franz Borkenau, militant autrichien qui avait joué un rôle dans le KPD des années 1920 mais avait violemment rompu avec le communisme, chercheur à l'Institut für Sozialforschung, publie dans ces deux années du pacte germano-soviétique, à Londres où il s'est réfugié, au début de 1940, The Totalitarian Enemy. ${ }^{32}$ Son livre, anticipant sur les thématiques de la Guerre froide, fait du communisme et du nazisme (le cas de l'Italie ne le retient guère) deux formes apparemment en conflit jusqu'alors, mais fondamentalement semblables de croyances eschatologiques et millénaristes. Les deux systèmes politiques, opposés en tout point à la démocratie, aux «liberal powers», aux droits et libertés qu'elles procurent et garantissent, sont fondamentalement les mêmes, prétend-il démontrer: planification économique, obéissance aveugle au Chef, propagande omniprésente, terreur policière les unit. Le contexte de 1939-41 fait que Borkenau n'a pas à prendre des gants, le Pacte vient de démontrer «au monde» que le nazisme en tant que rempart contre le

29 W. Gurian, Bolschewismus als Weltgefahr. Luzern: Vita nova, 1935. > Le bolchevisme, danger mondial. Paris: Alsatia, 1936. 59.

30 J. P. Faye, «Carl Schmitt, Jünger, Heidegger : le nazisme des intellectuels», Le Monde, 2 août 2013. Lire, de J.-P. Faye, L'État total selon Carl Schmitt, Ou comment la narration engendre des monstres. S. L. : Germina, 2013.

31 New York: Basic Books, 2014. Voir aussi, non moins récent, car le Pacte a été longtemps négligé des historiens, Robert Gellately, Stalin's Curse : Battling for Communism in War and Cold War. New York: Vintage Books, 2013. Notamment 49- .

32 London: Faber, 1939.

20 - Conexão Letras, Porto Alegre, v. 14, n. 22, p. 9-35, jul-dez. 2019. 
communisme était un mensonge de propagande. Le nazisme est un bolchevisme «brun» tandis que le communisme est un «fascisme rouge». Hitler est un Staline, peut-être en plus dangereux. Pour Borkenau, les analogies s'expliquent notamment dans la mesure où les mouvements et les régimes totalitaires «de droite» ont copié le brutal régime russe jusque dans le moindre détail et l'ont simplement transposé en clé réactionnaire. ${ }^{33} \mathrm{On}$ note une fois de plus que la thèse de la convergence imitative et «réactive» des totalitarismes, attribuée beaucoup plustard à Ernst Nolte, n'a pas attendu l'historien berlinois. Tout est esquissé avant 1945.

Borkenau conclut que la Guerre qui s'engage va être un affrontement ultimement idéologique entre des valeurs civiques et morales incompatibles, vouées dès lors à une lutte à mort. La survie de la civilisation dépend de l'issue de cette guerre. ${ }^{34} \mathrm{Il}$ faut distinguer les apparences des conflits et la réalité profonde, insiste-t-il: «The Nazi and the Bolshevist revolutions [are] only two specimens of one and the same movement, different in many secondary respects but one and the same in essence». ${ }^{35}$ Pendant les années de guerre, Borkenau écrit pour la revue Horizon. Dans ses nombreux articles, le nazisme est défini comme un fait avant tout religieux, comme la «wildest outburst of secular messianism ever experienced.» ${ }^{36}$ Le credo de ce messianisme racial est que «the Germans are God's Chosen people, by nature superior to all other people, predestined to rule the world and to bring salvation to it». Hitler est le Messie choisi par Dieu pour guider l'Allemagne vers l'hégémonie mondiale. Borkenau applique aussi le concept de messianisme au marxisme bolchevik. On a pu se demander, dit-il, comment une théorie rationaliste comme le marxisme avait pu s'emparer des masses russes. Les fondements messianiques du marxisme même et leur convergence avec le mysticisme russe orthodoxe procurent, selon lui, une explication. ${ }^{37}$ Borkenau fait dès lors de Karl Marx la source initiale du retour inopiné du messianisme dans la modernité séculière, contaminé ensuite de religiosité populaire russe. ${ }^{38} \mathrm{Il}$ n'y a guère de doute encore que Raymond Aron, à Londres lui aussi où il dirige la revue gaulliste La France libre, a eu connaissance de ce livre qu'il ne cite pas, et de ces articles, et s'en est inspiré pour son fameux essai de 1944 sur «L'avenir des religions séculières»». ${ }^{39}$

William Henry Chamberlin, journaliste et historien américain $(† 1969)$ est l'auteur en 1937 d'un autre réquisitoire contre le régime soviétique et contre le principe même de la propriété collectiviste, principe qu'il montre inscrit dans l'histoire longue du socialisme et de la pensée utopique en Europe, Collectivism: A False Utopia. ${ }^{40}$ Chamberlin publie ensuite en 1942 The World's Iron Age qui, en ceci également préfigurant la thèse d'Ernst Nolte, articule expressément l'idée d'un «Lien causal» entre bolchevisme et nazisme et met dès lors le léninisme à l'origine du malheur du siècle — faisant des fascismes des imitations réactionnaires de la révolution russe, engendrant des régimes concurrents dans

33 The Totalitarian Enemy, 209.

34 Préface datée de décembre 1939 à The Totalitarian Enemy, 11-13.

35 ibid., 197.

36 The Totalitarian Enemy, 129.

37 The Totalitarian Enemy, 127.

38 Ibid.

39 Réédité plus d'une fois, notamment dans Une histoire du XXe siècle. Paris: Plon, 1996. 141-222. Voir au volume II.

40 Collectivism: A False Utopia. New York: Macmillan; London: Duckworth, 1937. > Dépouillé sur la rééd. de 1938. 
l'inhumanité croissante de leur «rivalité mimétique» ${ }^{41}$ William Henry Chamberlin avait séjourné à la fois en URSS et en Allemagne, il pouvait comparer de première main et mesurer les influences exercées par Moscou.

Sigmund Neumann, autre politologue américain d'origine allemande, dans Permanent Revolution, essai publié à New York cette fois en $1942,{ }^{42}$ amorce une interprétation globale qui sera également retravaillée par les générations d'historiens jusqu'à nous: le «totalitarisme», tant bolchevik que nazi, est le résultat d'un vain effort de perpétuer la révolution, une révolution chimérique, impossible à réaliser, engendrant une fuite en avant dans des politiques de plus en plus volontaristes et répressives au fil des échecs et des résistances. Au contraire de Borkenau, Permanent Revolution observe toutefois de grandes différences entre nazisme et stalinisme qui n'ont vraiment de commun que le rejet de la démocratie, de l'état de droit et de l'individualisme. Le rejet du capitalisme, qui est total en Russie, se limite à une économie enrégimentée par l'État totalitaire en Allemagne.

Ludwig Von Mises enfin, économiste libéral autrichien, naturalisé américain, publie à la fin de la guerre Omnipotent Government : The Rise of Total State and Total War. ${ }^{43}$ Il regroupe lui aussi sans hésiter sous le vocable commun de «socialisme» les régimes bolcheviks et nazis. Les nazis sont des crypto-communistes! Ils ne maintiennent que les apparences de la propriété privée, les entreprises allemandes sont gérées par des Betriebsführers, il n'y a plus de marché du travail, le gouvernement fixe les prix et les salaires: le nazisme est donc bel et bien un socialisme. Dans les années de guerre, et particulièrement entre 1939 et 1941, Mises n'est pas seul, les libéraux de pure doctrine argumentent l'horreur que leur inspire le système nazi en se représentant les nazis comme des sortes de «marxistes», des bolcheviks dévoyés et déguisés en conservateurs pour amadouer l'ancienne classe dirigeante. Ludwig von Mises croyait observer en tout cas au début de la guerre que le nazisme s'était mis à appliquer la plupart des mesures préconisées par le Manifeste du Parti communiste. ${ }^{44}$

Tous ces livres en langue anglaise ont connu, avant et au cours de la guerre, un certain succès dans le monde savant et lettré ; ils confirment que la catégorie de totalitarisme, catégorie qui servait d'emblée à subsumer le nazisme et le bolchevisme, a été abondamment travaillée avant 1945 dans des essais qui ont connu une large diffusion. La Guerre froide ne fera que réveiller, prolonger et rediffuser dans un nouveau contexte ces sortes d'analyses.

\section{«Totalitarisme» dans la Guerre froide}

La locution même de «Guerre froide» est une expression datée, un oxymore tant soit peu hyperbolique, un instrument rhétorique et propagandiste. Elle est toutefois adoptée par un peu tout le monde pour désigner le laps de temps 1947-1988. Je l'utilise, mais

41 Chamberlin, The World's Iron Age. New York: Macmillan, 1942. Chamberlin publiera en 1949 son intéressante autobiographie, The Confessions of an Individualist.

42 Neumann, Permanent Revolution. The Total State in a World at War. New York, London: Harper, 1942.

43 New Haven CT: Yale UP, 1944 - Ce livre a été réédité en 1969, Grove City PA: Libertarian Press. > Cf. de L.v. M. Theory and History. London: Cape, 19571; Planned Chaos. Irving on Hudson: Foundation for Economic Education, 1972 [1947].

44 Omnipotent Government, The Rise of the Total State and Total War. New Haven: Yale University Press, 1944. 
elle vaut ce qu'elle vaut. Sobre de caractère et de style, Raymond Aron préférait parler de «paix belliqueuse» (avec quelques épisodes «chauds» mais limités et contenus par la prudence des antagonistes), état de chose qui a duré quarante et un ans. La Guerre froide répond tout d'abord à la mise en place, clé en main, prêts à l'emploi, sous l'égide de l'Armée rouge et du NKVD et sans les cruels tâtonnements qu'avait connus l'URSS d'avant-guerre, de régimes totalitaires servilement calqués sur le modèle stalinien en Allemagne de l'Est, en Pologne, Hongrie, Tchécoslovaquie, Albanie, Bulgarie et Roumanie. Un «rideau de fer» tombe sur l'Europe et la divise, il la coupe en deux pour un demisiècle. ${ }^{45}$ Sans parler des pays annexés par l'URSS avec l'aide du Reich allemand, Estonie, Lituanie, Lettonie, Moldavie qui ne recouvreront la souveraineté qu'en 1989-1990.

La notion de «régimes totalitaires» s'applique à coup sûr jusque 1956 et elle se perpétue chez les observateurs, avec des aménagements significatifs, au-delà du Rapport Khrouchtchev et du bref Dégel. L'instauration partout en Europe centrale de sous-cultes des secrétaires généraux est par exemple un argument en faveur du recours à la catégorie. J'ai évoqué, dans Le siècle des religions séculières, ces cultes calqués sur le culte stalinien qui prolifèrent dans l'Europe dite «de l'Est» des années 1950-60, nommément ceux de Màtyàs Ràkozi, Georgi Mikhailov Dimitrov, Walter Ulbricht, Klement Gottwald, $\mathrm{Bo}^{3} \mathrm{es}^{3} \mathrm{aw}$ Bierut, Gheorghiu Dej, Enver Hodja : même adoration obligatoire et lancinante de tout un peuple, mêmes anecdotes pieuses démontrant l'omniscience et l'infaillibilité et indéfiniment répétées, mêmes statues monumentales, mêmes poèmes sirupeux et adulateurs et œuvres d'art glorificatrices. Dans tous les cas, dans tous ces pays, ce sont des tentatives conscientes et organisées, intrinsèquement absurdes, de créer du charisme et de l'adoration de masse autour de leaders fort peu dotés par leur nature bureaucratique d'une quelconque aura poétique, d'attraits humains et de séduction personnelle.

C'est toutefois pendant la Guerre froide que le mot-notion de «totalitarisme» subit une mutation préjudiciable à un usage serein et rigoureux: il devient un mot d'ordre anticommuniste. Son emploi public propagandiste va peser sur les efforts d'interprétation politologique, sur les travaux savants et philosophiques qui devront chercher à s'en dépêtrer, à se dégager de sommaires instrumentalisations. En effet, le mot de «totalitarisme» en vue de désigner en les dénonçant les régimes sous hégémonie soviétique n'est pas en premier lieu, dans les années 1950 à 1980, un concept théorique, et sûrement pas au cours des années «chaudes» de la Guerre froide. Il est avant tout un mot brandi par les politiques, du moins dans les pays de langues germaniques et anglo- saxons. Dans un speech fameux, le président Harry Truman en 1947 déclare que les Américains doivent se tenir prêts «to help free peoples to maintain their free institutions and their national integrity against aggressive movements that seek to impose upon them totalitarian regimes.») C'est l'axiome de ce qui sera connu comme la Doctrine Truman. Le futur président Dwight Eisenhower utilise abondamment le terme dénonciateur pendant la campagne électorale de 1952: il déclare son intention d'aller en Corée et de mettre fin à la guerre engagée par les communistes dans ce pays. Le rapprochement - tyrannie pour tyrannie - avec la guerre victorieuse à laquelle le général Einsenhower a contribué, celle des démocraties (alliées à l'URSS) contre l'Axe est explicite dans son discours. La lutte continue car c'est un combat spirituel, une «croisade» contre le totalitarian mind. Autrement dit les penseurs académiques, les politologues et les historiens des années 1950-60 s'emparent, en vue cen-

45 L'expression apparaît dans le Discours prononcé par Winston Churchill à Westminster College le 5 mars 1946 alors que beaucoup considèrent encore les Soviétiques comme de fidèles et héroïques alliés. 
sément de lui conférer un sens serein et objectif, d'un mot instrumentalisé et sur-utilisé qui sert à désigner avec réprobation l'ancien allié devenu un adversaire, à construire ce qu'on sait de son régime en une hideuse antithèse des régimes «occidentaux» idéalisés, fondés sur le scrupuleux respect des droits et des libertés. Le mot est devenu polémique, il est dénégateur également ; il sera difficile de le débarrasser de cette charge quoique plusieurs s'y essayeront avec de variables succès et une variable indépendance d'esprit.

Il serait par contre fallacieux de réduire la réflexion philosophique et académique qui se développe à cette époque face à un monde coupé en deux, aux seuls usages propagandistes de «totalitarisme», accolé, pour le dénoncer d'un seul mot, à l'ancien allié, à l'URSS et à ses «satellites». Dans les polémiques historiennes depuis 1945, bien ancrées à droite et à gauche, inconciliables puisqu'il ne s'agit pas de distinguer le vrai du faux, le soutenable du mensonger, mais de «choisir son camp» (expression inopportune dans ce contexte), «totalitarisme» passe pour une catégorie qui n'est propre qu'aux historiens libéraux (donc «de droite»), catégorie qui rapproche les dictatures «totales» de droite et de gauche au grand dam de ceux qui gardent espoir dans l'avenir sinon le présent de l'URSS, — tandis que «Fascisme» est la catégorie qui montre inconciliables les deux systèmes, à la fois dans leur affrontement mais aussi en termes de comparaison, les régimes fascistes étant issus du nationalisme belliciste contre lequel tout d'un tenant ont combattu depuis les années 1930 les «antifascistes» groupés autour de l'URSS et du «Camp de la paix». Tout rapprochement URSS/Allemagne nazie - spontané et fréquent jusqu'en 1941 - est alors à répudier comme une obscénité: il ne peut être que le fait d'impérialistes, d'«anticommunistes viscéraux».

- Le maccarthysme : On a décrit à bon droit l'épisode du maccarthysme comme une dynamique totalitaire réactive de la droite américaine alimentée par les peurs des premières années de la Guerre froide. Cet épisode, on le sait, a été qualifié aussi, fort pertinemment dans le pays des sorcières de Salem, de «Chasse aux sorcières», Witch hunt. Cet épisode s'étend en gros de 1950 à 1954 ; il se termine avec un vote de censure du Sénat qui met fin au zèle excessif de Joseph McCarthy. Pendant deux ans (19531954), la commission d'enquête sur les «activités anti-américaines», Un-American Activities Committee, présidée par le fanatique sénateur traque d'éventuels espions de Staline, les militants et sympathisants communistes aux États-Unis, notamment dans les milieux artistiques et intellectuels, dans une atmosphère d'hystérie anticommuniste, de paranoïa, de délation, de chantage, de terreur. Cette hystérie mccarthyste s'étendait du reste, il faut le reconnaître, à une large part de l'opinion américaine.

\section{4 d'Orwell}

Quelques mois avant sa mort précoce, l'écrivain britannique George Orwell voit publier son roman Nineteen eighty-four: A Novel. London, New York: Harcourt, Brace, 1949. Cet ouvrage dystopique s'impose immédiatement comme un best-seller, 400000 exemplaires sont vendus dans l'année même en Angleterre et aux Etats-Unis seulement. Conspué par la gauche conformiste comme une «opération anti-communiste», le roman devient l'enjeu d'une violente bataille idéologique. Orwell qui a une excellente connaissance des livres sur les totalitarismes dont je viens de faire état et des écrits des opposi- 
tionnels sur le Pays du Grand mensonge ${ }^{46}$ voulait avant tout faire une satire des idées et des langages totalitaires, de la destruction de la langue à coup d'antiphrases, perversion entraînant celle de la rationalité et des valeurs morales, avec les Semaines de la Haine, les Maisons de la Victoire, la Ligue Anti-Sexe Juniors, le Ministère de l'Amour, et surtout le Ministère de la Vérité, chargé de répandre le mensonge d'État. La satire inspirée à la fois par le nazisme et par l'URSS est amère: «Le Parti finirait par annoncer que deux et deux font cinq et il faudrait le croire. Il était inéluctable que, tôt ou tard, il fasse cette déclaration. La logique de sa position l'exigeait. Ce n'était pas seulement la validité de l'expérience, mais l'existence même d'une réalité extérieure qui était tacitement niée par sa philosophie. L'hérésie des hérésies était le sens commun. $\rangle^{47}$

On relie la genèse psychologique de $1984 \mathrm{chez}$ Orwell à son expérience traumatique de la Guerre d'Espagne, à son observation du rôle abominable des hommes du Komintern. Le Komintern qui manipule la catégorie «antifasciste» à sa guise, mobilise les progressistes dans la Guerre d'Espagne tout en liquidant en sous-main les oppositionnels et en dénonçant comme «fasciste» quiconque exprime des doutes sur les Procès de Moscou. Orwell observe que les Soviétiques, censés soutenir les Brigades internationales, s'activent surtout en Catalogne à éliminer les anti-staliniens, les militants du POUM, le Partido Obrero de Unificación Marxista, et à liquider les anarchistes. Le totalitarisme en action, c'est la négation de l'histoire, à tout le moins, sa suspension effective et délibérée. Orwell en eut la première intuition lors de la guerre d'Espagne ; et l'on peut voir dans la révélation qu'il eut alors comme le premier germe de 1984. Il en fit la réflexion à Arthur Koestler, qui avait partagé cette même expérience: «L'Histoire s'est arrêtée en 1936.» «Ainsi, la propagande stalinienne effaca toutes traces de batailles gagnées par les républicains lorsqu'il s'agissait de milices anarchistes et inventa de grandes victoires communistes là où nul combat n'avait été livré. Dans la presse communiste, l'expérience du front qu'avaient vécue Orwell et ses camarades se trouva frappée de totale irréalité. L'exercice du pouvoir totalitaire ne peut tolérer l'existence d'une réalité historique.» ${ }^{48}$

Le Britannique Aldous Leonard Huxley est connu pour son roman Le Meilleur des mondes. Brave New World est un roman d'anticipation dystopique, écrit en 1931, publié en 1932. Vingt-trois ans plus tard, en 1954, Huxley a publié non une fiction mais un essai de prospective, Brave New World Revisited. Le Retour au meilleur des mondes prétend montrer, vingt-deux ans après, une évolution accélérée des sociétés modernes qu'il perçoit comme courant dangereusement vers le monde cauchemardesque décrit dans son récit d'avant la guerre. Huxley cherche en somme à faire admettre que la vision d'une société inhumaine centrée sur les totalitarismes communiste et fascistes de son «concurrent» George Orwell était partielle et unilatérale. Certes, en tant que société punitive, répressive et policière, les totalitarismes (il emploie le mot) se sont distingués dans l'horreur, mais l'URSS se déstalinise en 1954 tandis que les États des sociétés dites

46 Je me rapporte au titre fameux d'Ante Ciliga, Dix ans au pays du mensonge déconcertant. Éd. définitive. Paris: Champ libre, 1977. Éd. originale partielle, 1938, Au pays du Grand mensonge > Rééd. 1950.

47 George Orwell, 1984, trad. A. Audiberti, Paris: Gallimard « Folio »,1991, 118. Homage to Catalonia de George Orwell raconte la Guerre civile espagnole et dénonce les crimes des agents staliniens aux dépens des autres républicains. La traduction française n'est publiée qu'en 1955 aux éditions Gallimard sous le titre $L a$ Catalogne libre.

48 «Orwell, selon Simon Leys», Le Figaro, 02/11/2006. 
démocratiques, alliés au pouvoir du Grand capital «corporate», lui apparaissent tentés de recourir à des moyens psycho-techniques doux, plutôt qu'à la violence nue, pour contrôler et conformer les populations qu'ils administrent. C'est un plaidoyer pro domo: Huxley pense que, l'épisode totalitaire stalinien-nazi étant en voie d'être dépassé, c'est son Brave New World avec son pessimisme global et sa crainte, non de frustres et sanguinaires dictatures de gauche et de droite, mais de l'État «panoptique» faussement bienveillant, qui avait vu juste à moyen terme. Orwell, à son gré, était un optimiste! Huxley est, lui, un pessimiste prospectif radical. Il lui semble en premier lieu que la démographie planétaire, l'accroissement asymptotique de la population auquel il consacre le Ier chapitre recèle en soi des dangers auxquels les États dans l'avenir ne pourront faire face qu'en sacrifiant la démocratie et les libertés. Il voit une corrélation étroite «entre un nombre trop grand d'hommes qui se multiplient trop rapidement et l'énoncé de philosophies autoritaires, l'apparition de systèmes totalitaires de gouvernement.» ${ }^{49}$ Le véritable totalitarisme, in-déboulonnable, est devant nous, on n'a encore rien vu. «Les anciens dictateurs sont tombés parce qu'ils n'ont jamais pu fournir assez de pain, de jeux, de miracles et de mystères à leurs sujets; ils ne possédaient pas non plus un système vraiment efficace de manipulation mentale. ... Sous la férule d'un dictateur scientifique, l'éducation produira vraiment les effets voulus et il en résultera que la plupart des hommes et des femmes en arriveront à aimer leur servitude sans jamais songer à la révolution. Il semble qu'il n'y ait aucune raison valable pour qu'une dictature parfaitement scientifique soit jamais renversée. ${ }^{50}$

\section{Le classique de la Guerre froide, Totalitarian Dictatorship and Autocracy}

C'est en 1956 que deux politologues américains, Carl Joachim Friedrich et Zbigniew Brzezinski publient le livre qui sera le classique académique de la Guerre froide vue par le «Monde libre», Totalitarian Dictatorship and Autocracy. Leur livre paraît dans les mois de l'invasion de la Hongrie qui semble confirmer, par delà la mort de Staline, leurs analyses et leurs pessimistes conjectures. ${ }^{51}$ Les deux politologues élaborent une définition par paramètres cumulatifs, donnés pour suffisants et nécessaires, qui sera indéfiniment répétée dans des centaines de manuels de science politique anglo-saxons.

Les paramètres retenus par Friedrich et Brzezinski comme nécessaires et suffisants à l'idéaltype sont six, à savoir:

- une idéologie «totaliste» d'État (l'adjectif «totaliste» lui-même n'est pas vraiment défini - et comme il est redondant avec le definiendum, le tout apparaît d'emblée tautologique.) Carl Friedrich en vue de suggérer un caractère propre et commun aux idéologies communiste, fasciste et nazie, parlera un peu plus tard d'idéologies «chiliastiques» (synonyme en grec de «millénariste»), mais ce mot d'origine religieuse, inspiré par Norman Cohn sans doute, soulève un immense problème de persistance historique sur lequel la définition fait l'impasse;

49 Ibid.

50 Ibid., § final.

51 J'ai dépouillé Totalitarian Dictatorship and Autocracy sur la 2nd Ed. rev. Cambridge: Harvard UP, 1965 [Originale: 1956]. 
- un parti unique, à structure rigoureusement hiérarchique, voué à l'application de cette idéologie,

- une police secrète pleinement développée;

- trois monopoles d'État:

- celui des communications,

- celui des armes, et la mainmise sur toutes les organisations civiles, spécialement économiques. ${ }^{52}$

Friedrich et Brzezinski aboutissaient, découlant de leur idéaltype, à une hypothèse prédictive, — or, c'est elle qui a été réfutée par le cours des choses, par l'histoire de l'URSS de 1956 jusqu'à sa dissolution: l'inférence «logique» de la définition était que de tels régimes sont irréformables (ce qui peut demeurer «vrai» si on considère que les réformes un jour entreprises les déstabilisent, mais ceci pourrait se dire de tout régime en crise). Les Cold Warriors pensaient en fait que les totalitarismes ne peuvent jamais ni s'adoucir ni s'édulcorer. L'évolution de l'URSS et son effondrement final ont montré à tout le moins que cette conjecture n'a pas été confirmée, s'il est vrai que l'on peut soutenir qu'il y a une limite à la «libéralisation» au-delà de laquelle un système post-totalitaire se disloque.

\section{Hannah Arendt et la déchéance irrationnelle de l'Occident}

J'en viens - pour terminer sans conclure - au livre profond, intense, plein d'idées perspicaces, mais aussi lacunaire et contradictoire, The Origins of Totalitarianism de Hannah Arendt achevé en 1949 et dont le premier volume sort en février 1951. ${ }^{53}$ La trilogie d'Arendt est peu cohérente à commencer par son plan général: — la première partie, sur l'antisémitisme, ne traite que des origines idéologiques du nazisme (Arendt exclut de son enquête l'Italie fasciste où subsistaient l'Église, la monarchie etc.); — la deuxième porte sur les «origines» dans l'impérialisme européen et le racisme d'un totalitarisme réduit ici encore au seul nazisme. À ce titre, Arendt entrait en conflit frontal avec l'antitotalitarisme simplet de la Guerre froide comme idéologie d'un «Monde libre» qui eût été innocent du malheur du siècle. La troisième partie enfin traite «phénoménologiquement» du totalitarisme comme d'une forme nouvelle et inouïe de régimes politiques, régimes absolument distincts de toutes les formes de tyrannie passées. Elle en traite en confrontant les deux systèmes nazi et soviétique duquel il n'avait guère été question dans les volumes précédents. Mais ici, Arendt ne traite plus ou guère de ses «origines», de leurs éventuelles origines communes et la comparaison même tourne court.

Arendt avait entrepris la rédaction des Origins au bout du huit années d'une vie de réfugiée politique aux États-Unis, au milieu d'autres exilés et émigrés européens, surtout des Allemands. C'est à la fin de ces années qu'Arendt prend peu à peu conscience du réseau de camps et du régime de terreur prévalant en URSS et de ses terribles analogies avec le totalitarisme nazi. Peut- être que la chasse aux sorcières du sénateur McCarthy

52 Friedrich, Carl Joachim et al. Totalitarianism in Perspective: Three Views. New York: Praeger, 1969, 126. On verra aussi : Brzezinski, Zbigniew. The Grand Failure: The Birth and Death of Communism in the 20th Century. New York: Scribner, 1989.

53 Avec d'autres écrits : Les origines du totalitarisme. Eichmann à Jérusalem. [...] Éd. Pierre Bouretz. Paris: Gallimard, 2002. 
qui battait son plein lors de la sortie du livre a incité Arendt à tempérer sa dénonciation du système stalinien et du moins à chercher à rendre ses analyses bien distinctes de l'anticommunisme grossier de la droite américaine et de celui des anciens communistes «renégats» qui n'hésitaient pas, au nom de la démocratie à laquelle ils s'étaient «convertis», à recourir à des moyens «proto-totalitaires» pour lutter contre l'idéologie reniée.

L'Idée de départ de la philosophe est que les grands types de régimes politiques dans les sociétés ont été très peu nombreux et ont été recensés et décrits depuis la plus haute antiquité. La plupart se sont avéré d'une extraordinaire longévité. Le totalitarisme est alors une nouveauté à l'horizon de la condition humaine, il est un événement sans précédent, l'apparition d'un mal radical, inconnu des hommes auparavant et inconcevable par eux. Pas seulement une nouveauté politique, mais des régimes où les catégories politiques et morales léguées par les siècles ne s’appliquent plus. «L'institution de régimes totalitaires, dans la mesure où leurs structures et leurs techniques sont absolument sans précédent, représente la nouveauté essentielle de notre époque. En comprendre la nature - ce qui n'est possible qu'après avoir décrit et analysé les origines et les structures du phénomène - équivaut pour ainsi dire à comprendre le cœur même de notre siècle.»

Loin de faire du totalitarisme, à la façon de Friedrich et Brzezinski, un idéaltype statique, Arendt cherche à éclairer la genèse du phénomène, à l'expliquer par ses «origines». Sa question n'est pas qu'est-ce que c'est?, mais comment cela a-t-il été rendu possible? Sa réponse est puissante : le totalitarisme est le chemin de traverse pris par toute la civilisation occidentale, il est issu d'un effondrement moral de celle-ci. ${ }^{54}$ Arendt aboutit à une mise en accusation d'un 19e siècle, première phase de la modernité, où les idées et les projets totalitaires trouvent leur origine avant le passage à l'acte au siècle suivant. La naissance de la société de masses apathiques et mobilisables, l'anomie croissante des foules sont au cœur de sa réflexion, comme la décomposition alléguée de l'État-nation, les progrès de l'impérialisme (éclectique, Arendt emprunte au marxisme ici qu'elle combine à Heidegger et au Kulturpessimismus) et la mégalomanie des Grandes politiques issue de la fusion du capitalisme conquérant et des égoïsmes nationalistes, l'expansionnisme européen, le colonialisme, le social-darwinisme, fondement du «droit à la domination» et venant corrompre la démocratie des métropoles. L'antisémitisme, le pan- germanisme, le pan-slavisme sont donnés pour les éléments originels convergents des futures pratiques totalitaires. La genèse du fait totalitaire en Occident entre 1884 et 1914 tient dès lors aux progrès irrépressibles d'une irrationalité mondialisante, d'une mégalomanie de politiques expansionnistes, d'une déraison croissante de toutes les sociétés occidentales; à la déraison des élites se soumettent des foules percluses de ressentiment et de chauvinisme, non moins que les cyniques agents d'un capitalisme expansionniste. (C'est l'approfondissement de la thèse soutenue par les socialistes et les anarchistes d'avant 1914, que l'expansion coloniale impérialiste aboutirait fatalement à corrompre le peuple et à dégrader la démocratie en métropole).

Arendt s'évertue donc à chercher des causes à l'émergence des machineries de domination totale, mais les causes qu'elle discerne ne parviennent pas, elle l'avoue, à expliquer intégralement l'irrationalité essentielle du totalitarisme, son défi à toutes les lois établies y compris les siennes propres: l'État détenteur des fins ultimes de la société, la

54 On verra aussi: Arendt, Understanding and Politics. On the Nature of Totalitarianism. > La nature du totalitarisme, trad. M.-I. Brudny de Launay. Paris: Payot, 1990. Rééd. Payot-Rivages, 2006. 
terreur comme essence du régime, la politique émancipée de tout scrupule moral, les «mensonges énormes», les contre-vérités sur lesquels ces régimes reposent, le secret ubiquitaire (les institutions censées «légales» n'ont jamais le pouvoir qu'on leur attribue «sur papier»), la volonté de continuer à faire peser la terreur sur une population entièrement soumise, les purges récurrentes, la liquidation de groupes et de couches sociales qui ne sont même pas ennemis du régime, la totale indifférence à l'efficacité, notamment économique, la «gabegie planifiée» en dépit d'une gigantesque inflation d'un appareil bureaucratique plein de conflits et de dédoublements, la substitution, en fin de compte, de l'idéologie à la réalité.

Hannah Arendt aboutit à définir le totalitarisme dans les termes suivants: il s'agit d' «une forme de gouvernement dont l'essence est la terreur et dont le principe d'action est le caractère logique de la pensée idéologique». Les notions séculaires d'innocence et de culpabilité, de droit, de légitimité, de légalité sont effacées au profit de la terreur d'État. Dans un article de 1953, "Ideology and Terror", Arendt revient sur la rupture morale radicale qu'ont opéré à la fois le Troisième Reich et l'URSS : «Même le châtiment du criminel est encore un acte de responsabilité et de solidarité humaine. Les chambres à gaz du Troisième Reich et les camps de concentration de l'Union soviétique (qui sont également des camps d'extermination, même si les méthodes y sont différentes) ont rompu la continuité de l'histoire occidentale, car personne ne peut sérieusement assumer la responsabilité de telles entreprises, pas plus qu'on ne peut sérieusement en imputer la responsabilité à quiconque. ${ }^{55}$ L'essence du régime totalitaire se substitue à toutes les règles juridiques et morales millénaires. Si la légalité définit l'essence du gouvernement constitutionnel, c'est la terreur qui définit celle du gouvernement totalitaire. La terreur devient l'essence d'un gouvernement lorsqu'elle est dirigée contre des victimes qui sont innocentes, même du point de vue du régime persécuteur; d'importantes parties de la population sont punies non pour des crimes dont elles sont soupçonnées, mais pour ceux qu'elles pourraient commettre. Bolchevisme et nazisme : «Dans les deux cas, certains groupes, indépendamment de leurs opinions, de leurs actions et de leur comportement, se trouvent désignés comme les ennemis de l'humanité : dans un cas, la bourgeoisie, à titre d'ennemi historique qui, seul, fait obstacle à la victoire du prolétariat et, partant, à l'édification d'une société sans classes et à l'avènement de la justice, et, dans l'autre, les Juifs en tant qu'ennemis naturels de l'humanité, contre-race. $\rangle^{56}$ Dans ces pages et dans d'autres, le parallèle entre les régimes bolchevik et nazi est systématiquement développé.

Arendt qui dessine un débouché totalitaire de toute l'histoire européenne moderne, met en cause une convergence de dynamiques politiques et sociales perverses depuis la révolution industrielle. C'est comme la résultante de cette dynamique que le totalitarisme émerge en tant que phénomène inouï qui rompt avec tout le passé, avec l'histoire elle-même comme séquence d'événements plus ou moins marquants et intelligibles. Ce qui est puissant chez Arendt, et dérangeant, c'est que le totalitarisme, très différent de l'objet-repoussoir bricolé par l'idéologie de la Guerre froide, ce n'est pas les «autres», ce ne sont pas des extrémistes, pas de répréhensibles anti-démocrates et anti-libéraux,

55 «Ideology and Terror», The Review of Politics, 15/3, july 1953. 303-327; trad. Idéologie et terreur, introduction par Pierre Bouretz. Paris: Hermann, 2008. 53.

56 Arendt, Understanding and Politics. On the Nature of Totalitarianism. > La nature du totalitarisme. Trad. M.-I. Brudny de Launay. Paris: Payot, 1990. d Rééd. Payot-Rivages, 2006, 99. 
ce ne sont pas les seuls bolcheviks et fascistes, c'est sur quoi débouche toute l'histoire occidentale. Les «origines» des régimes de terreur et des génocides et massacres de masse du 20e siècle sont civilisationnelles; elles remontent à la fois à la révolution industrielle, à la mégalomanie des classes possédantes et à la naissance de la politique de masse en 1789 , - aux «despotisme de la liberté», «règne de la vertu», à la soumission à la Volonté générale, à la conscription et la levée en masse. (Le rapprochement qu'Arendt esquisse avec 1789- 1793, Robespierre /Lénine, Fouquier-Tinville/Vychinski, Terreur jacobine/ terreur stalinienne n'a rien d'original: c'est un lieu commun des historiens du 20e siècle).

Les sociétés de masse, ces magmas où sont dissous les individus, les classes et les rangs - type de sociétés issues de l'effondrement du système traditionnel des classes et de l'affaiblissement de l'État-nation - naissent avec les progrès mêmes de la démocratie et de l'État de droit mais elles recèlent un danger immanent pour ceux-ci. Ce sont des sociétés qui se sont peu à peu atomisées, où les hommes sont tentés par l'autoritarisme et travaillés par l'intolérance et la haine. Cette thèse fait penser à l'ambivalence de Tocqueville face à la démocratie. Les «générations du front» aventurières et nihilistes de 1918 figurent une version exacerbée de cette tendance. Les chefs totalitaires s'appuient sur des masses désorientées. Le crime et la violence exercent un puissant attrait sur elles. L'individu en manque d'insertion sociale s'identifie au Groupe que lui définit et désigne l'idéologie totalitaire. Les procès de Moscou, la liquidation des SA n'ont été possibles que parce que les masses approuvaient tacitement. "La tentative totalitaire de rendre les hommes superflus reflète l'expérience que font les masses modernes de leur superfluité sur une terre surpeuplée». ${ }^{57}$ Le lien entre atomisation sociale et totalitarisme est au cœur de la réflexion.

Arendt met le fait des camps - sans isoler dans une horreur à part les camps d'extermination des nazis - comme la preuve en acte et l'éminente illustration de cette idée de superfluité des humains. C'est précisément ce phénomène des camps, «la plus importante institution de la domination totalitaire» qui lui permet d'inclure nazisme et communisme soviétique dans une même conceptualisation.

Arendt donne à l'idéologie un rôle décisif, idéologie d'État toujours couplée à la terreur, - mais la dite terreur, «essence» du régime, est la conséquence du primat absolu de l'idéologie totale, du primat du «délire idéologique» substitué à l'expérience empirique. Bien entendu, ajoute-t-elle, «là où le règne de la terreur est porté à la perfection, comme dans les camps de concentration, la propagande disparaît complètement. ${ }^{58}{ }^{58} \mathrm{Ce}$ rôle fondateur et déterminant de l'idéologie, thèse qui préfigure le concept d'«idéocratie» chez un Martin Malia, c'est une idée originale et un élément trop peu souligné des Origins of Totalitarianism. Nous y retrouvons une interprétation forte du «vieux» problème du rôle des idées dans l'histoire.

Au tome III, Arendt montre la convergence du communisme bolchevik et du nazisme dans une semblable plongée dans l'inhumanité. Elle la constate mais elle reconnaît ellemême plus d'une fois qu'il est «curieux» («it is a curious fact») que, partant de principes économiques, idéologiques et culturels diamétralement opposés, les deux régimes en conflit ont abouti à «des résultats structurellement identiques». La réflexion de la philosophe ne cherche pas vraiment à lever cet «étonnement» qui demeure numineux et angoissant. L'analyse sur ce point essentiel tourne court. Idéologie et terreur définissent 
le totalitarisme: il semblerait alors que le contenu «diamétralement opposé» des idéologies, soit ait importé bien peu, ou bien qu'en dépit des apparences, les dites idéologies communiste et fascistes possédaient des traits communs et une essence idéelle semblable. Ce serait à mon sens l'alternative à trancher qu'Arendt ne creuse pas, bien que toute sa généalogie des Origines invite à conclure à l'inclusion des idéologies extrêmes en conflit dans une même matrice irrationnelle de la modernité occidentale. La question qui est tout de même fondamentale de savoir en quoi les idées qui nourrissent le nazisme/ fascisme aboutissent à engendrer aussi le totalitarisme soviétique n'est en tout cas pas explicitement posée - à moins de considérer, et c'est vers quoi mène à mon sentiment la généalogie arendtienne, l'ainsi nommé marxisme-léninisme comme un avatar, sous des habits socialisants et humanitaires, du productivisme et de l'étatisme. Telle était, l'idée suspicieuse et l'argument-clé des anarchistes et libertaires de la Belle époque opposés au cauchemardesque «collectivisme d'État» rêvé par leurs frères ennemis, les «socialistes autoritaires», ces prisonniers des idées «bourgeoises».

Arendt infère aussi que les totalitarismes sont des régimes, de par le grandiose et chimérique but final qu'ils poursuivent, en mouvement perpétuel, toujours agités, toujours résolus à re-mobiliser les masses pour quelque nouvelle étape de leurs Grandes politiques. Un régime totalitaire ne peut survivre que par la fuite en avant. Les idéologies soviétique et nazie, dissemblables en apparence, se rejoignent dans ce qu'Arendt désigne comme la «loi du mouvement» qui découle de leurs «idées» fondatrices, étant l'expression activiste du déterminisme historique dont elles sont imbues toutes deux.

Arendt attribue toutefois la totalitarisation de l'URSS au seul Staline et en exonère, sans argumenter non plus et sans citer aucun fait car elle ne connaît visiblement pas grand chose de la période, la dictature révolutionnaire de Lénine et la suite des années 1920. «Pour transformer la dictature révolutionnaire de Lénine en un régime complètement totalitaire, Staline fut d'abord obligé de créer artificiellement cette société atomisée que les circonstances historiques avaient déjà préparées en Allemagne pour les nazis». ${ }^{59} \mathrm{Ce}$ contraste est douteux. Arendt délimite son concept dans le temps. Il est possible qu'elle veuille sauver quelque chose de l'espérance révolutionnaire déçue par le cours de la réalité soviétique. Arendt évoque une sorte de coup d'état de Staline en 1929 qui serait la date a quo du passage au totalitarisme - coup d'état imaginaire dont les archives n'ont pas gardé de trace. Accablant Staline, Arendt demeure une sorte de léniniste ; Arendt ne se pose pas la question de savoir comment l'URSS serait passée d'une transitoire dictature révolutionnaire à un régime totalitaire, alors que «la politique suivie par Staline à partir de 1928-29 se contentait de reprendre et de systématiser celle inaugurée par son maître à penser et à agir dès 1917-18.» ${ }^{60}$ Elle exonère Lénine, «véritable homme d'État», elle rend hommage à Khrouchtchev et fonde quelque espoir en lui, et elle ne parle pas de Mao Zedong dont le régime mégalomane et meurtrier tourne pourtant alors à plein — et elle écarte de sa mire le régime qu'elle juge avoir été relativement bénin de Mussolini. Ici aussi, il est évident qu'elle n'a pas creusé la question de l'Italie.

Le totalitarisme, souligne la philosophe, est avant tout difficile à comprendre et le penseur ne doit pas vouloir dépasser cette difficulté au moindre prix ni refouler ce qu'elle a d'angoissant. Elle avoue qu'il lui demeure, au bout de ses efforts, en partie

59 Origins, 627.

60 Stéphane Courtois. Communisme et totalitarisme. Paris: Perrin, 2009. 
inintelligible. Il est l'impossible et l'impensable devenus possibles. En ce qui touche à l'irrationalité inhérente de ce qu'on ne peut platement appeler de «simples» régimes politiques, Arendt est perspicace, mais, dans la foulée, elle place la barre très haut pour procurer une explication tant soit peu satisfaisante à la mise en place de ce monde absolument nouveau où les hommes sont devenus «superflus». Le totalitarisme dépasse l'entendement humain, c'est en quoi il semble l'obliger à n'en pas faire un phénomène contingent et localisé, mais à reconnaître que toute la civilisation occidentale, ses valeurs et ses croyances sont en cause, qu'elles se sont préalablement effondrées. ${ }^{61}$ Arendt fait ainsi éclectiquement converger dans sa réprobation de la modernité - très heideggerienne en une transposition libérale (!), la modernité comme «Oubli de l'être» - la critique socialiste de l'expansion capitaliste et de l'impérialisme et la critique «de droite» à la Gustave Le Bon et à la Ortega y Gasset de l'irrationalité des masses, déboussolées et nihilistes, et celle de l'atomisation de la société - le tout pour aboutir à une condamnation englobante des dynamiques de la modernité.

Telle s'exprime la mise en accusation par une philosophe "sans attaches», une penseuse heimatlos, profondément pessimiste en dépit de bouffées d'optimisme occasionnel, de la modernité tout entière, impérialiste, productiviste, mais tout aussi bien celle de la modernité censée «progressiste» avec ses absurdes «lois de l'histoire» et leur fausse morale immanente, une modernité vue comme un processus de déraison et déshumanisation au bout duquel l'homme est devenu «superflu». Son humanisme philosophique nostalgique, basé sur les Grecs, les Romains et les anciens Juifs, le sentiment exprimé partout dans son œuvre d'un Never more, bien loin de tout optimisme d'une ré-institution morale et sociale à portée de main, - tout ceci, qui lui fait tirer philosophiquement tous azimuts contre toutes les politiques et institutions modernes, ne pouvait être mis au service d'aucun «camp» politique. Les gens de gauche qui auraient trouvé des arguments chez elle en cherchant un peu, n'ont pas lu Arendt, trop américaine et trop anti- soviétique, et les gens de droite, tout en se réclamant d'elle, n'ont pas voulu comprendre sa thèse «inutilisable» de la matrice formée par une civilisation occidentale en voie d'effondrement moral sous les coups de l'impérialisme, - thèse qui rendait dérisoire toute exaltation d'un libéralisme démocratique intemporel. Dans la conjoncture de la Guerre froide, c'est la disjonction même du totalitarisme et de l'impérialisme qui scelle l'idéologisation du concept à titre d'instrument polémique contre l'extrême gauche. Son réquisitoire qui met en cause toute la modernité «démocratique» n'empêchera toutefois pas son tortueux ouvrage, dûment simplifié et mis à plat, d'être instrumentalisé par les anticommunistes américains comme, lors de la traduction en allemand, par la majorité de droite d'Adenauer, posant en défenderesse du «Monde libre».

Les régimes totalitaires disparus étendent leur ombre sur le monde d'après leur chute. L'énoncé le moins pessimiste que l'on puisse repérer dans les écrits de la philosophe est quelque chose comme ceci: «le totalitarisme disparaîtra simplement un jour sans laisser d'autre trace dans l'histoire de l'humanité que des peuples épuisés, le chaos social et économique, le vide politique et une tabula rasa spirituelle. $\rangle^{62} \mathrm{C}^{\prime}$ est sur ce propos crépusculaire que se concluent les Origines. Hannah Arendt esquisse une morale stoïque de la bienfaisante dés-illusion: nous, Modernes tardifs, allons devoir finalement apprendre à vivre sans illusoires espérances, «in the bitter realization that nothing has been promised

61 Origines, 846.

62 Origines, 861.

32 - Conexão Letras, Porto Alegre, v. 14, n. 22, p. 9-35, jul-dez. 2019. 
to us, no Messianic Age, no classless society, no paradise after death». ${ }^{63}$ Elle épouse ici la vieille thèse rationaliste de la religion condamnée à terme qu'elle combine à celle des «religions politiques», nommément du marxisme, substituts archaïco-modernes des religions révélées ayant largement contribué au malheur du siècle, les millénarismes socialistes n'ayant été que l'avatar d'une Illusion dont l'humanité devait finir par se désabuser même si la lucidité cher payée devait être «amère» et pas seulement libératrice. L'idée que poursuit Arendt est celle du désenchantement comme nécessité éthique et comme ce processus historique entamé avec le scepticisme libertin et philosophique à l'égard des religions révélées qui devra, quoi qu'on en ait, s'accomplir jusqu'au bout. Tout à fait opposée aux sociologues conservateurs à la Vilfredo Pareto avec leur doctrine méprisante des religions, antiques et modernes, chrétiennes et socialistes, comme des impostures utiles se succédant indéfiniment les unes aux autres, Arendt soutient la thèse d'une dés-illusion nécessaire de l'homme moderne, devenu sobre et raisonnable, mais privé de promesse de bonheur et d'espoir de réparation des maux sociaux et tenu de regarder sans ciller un monde par bien des côtés insupportable.

Arendt s'est fait des ennemis en Europe et en Amérique avec ses trois volumes - et de plus coriaces encore, du côté sioniste comme du côté antifasciste, avec son ultérieur Eichmann à Jérusalem. Ses amis sionistes lui reprochèrent de montrer Israël reproduisant tous les travers de l'État-nation impérialiste. On lui a reproché aussi de ne pas confesser l'unicité de la Shoah. Son point de vue d'exilée et d'apatride qui n'épargne rien ni personne, qui est sans tendresse pour quelque fétiche que ce soit, a blessé plus d'un lecteur et nombre de ses amis. Si son œuvre a connu un vif regain d'influence et de prestige après la Chute de 1989-1991, c'est aussi que personne, et sûrement pas le «camp» libéral, n'a repris - ni pris au sérieux - sa condamnation tous azimuts de la modernité occidentale rongée par l'impérialisme et roulant à vau-l'eau.

\section{Références}

ARENDT, Hannah. Les origines du totalitarisme. Eichmann à Jérusalem. Éd. Pierre Bouretz. Paris: Gallimard, 2002. . As origens do totalitarismo. São Paulo: Companhia das Letras, 1989.

. La nature du totalitarisme. Paris: Payot, 1990. Trad.: M. I. Brudny de Launay. Réed. Payot- Rivages, 2006.

ARON, Raymond. Une histoire du XXe siècle. Paris: Plon, p. 141 - 222, 1996.

BERGOUGNIOUX, Alain; GRUNBERG, Gérard. L'utopie à l'épreuve, le socialisme européen au $20^{\text {e }}$ siècle. Paris: De Fallois, p. 95, 1996.

BORKENAU, Franz. The Totalitarian Enemy. London: Faber, 1939.

BROSSAT, Alain. L'épreuve du désastre: le 20e siècle et les camps. Paris: Albin Michel, 1996.

BRUNETEAU, Bernard. Le siècle des génocides. Paris: Colin, 2004.

BRZEZINSKI, Zbigniew. The Grand Failure: The Birth and Death of Communism in the 20th Century. New York: Scribner, 1989.

CHAMBERLIN, William H.. Collectivism: A False Utopia. New York: Macmillan, 1937.

63 The Origins of Totalitarianism, 436. 
. Collectivism: A False Utopia. London: Duckworth, 1937.

. The World's Iron Age. New York: Macmillan, 1942.

CHAUNU, Jean. Le paradigme totalitaire. Paris: F-Xavier de Guibert, p. 16, 2009.

CILIGA, Ante. Dix ans au pays du mensonge déconcertant. Paris: Champ Libre, 1997.

COURTOIS, Stéphane. Communisme et totalitarisme. Paris: Perrin, 2009.

CUMIN, David. Carl Schmitt, Biographie politique et intellectuelle. Paris: Cerf, 2006.

FAYE, Jean-Pierre. Langages totalitaire. Paris: Hermann, 1972. août, 2013.

. "Carl Schmitt, Jünger, Heidegger : Le nazisme des intellectuels". Le Monde, 2

. L'État total selon Carl Schmitt, Ou comment la narration engendre des monstres.

S.L: Germina, 2013.

FRIEDRICH, Carl J.; BRZEZINSKI, Zbigniew. Totalitarian Dictatorship and Autocracy. Cambridge: Harvard UP, 1965.

FRIEDRICH, Carl J. et al. Totalitarianism in Perspective: Three Views. New York: Praeger, p. 126, 1969.

FROMM, Erich. Escape from freedom. New York: Farrar \& Rinehart, 1941. Réed. sous le titre The Fear of Freedom, London: Routledge \& Kegan Paul, 1942.

GELLATELY, Robert. Stalin's Curse: Battling for Communism in War and Cold War. New Work: Vintage Books, 2013.

GENTILE, Emilio. Pour ou contre César? Les religions chrétiennes face aux totalitarisme. Paris: Aubier, p. 146, 2013.

GENTILE, Giovanni. "Fascismo (dottrina del)", Enciclopedia Italiana. Roma: Istituto dell'Enciclopedia Italiana, 1932, vol. XIV, p. 835-840.t

GUILHAUMOU, Jacques. Discours et événement: l'histoire langagière des concepts. Besançon: PU de Franche-Comté, 2006

GURIAN, Waldemar. Der Bolschewismus: Einführung in Geschichte und Lehre. Freiburg iB: Herder, 1931.

UP, 1952.

. Bolshevism: An Introduction to Soviet Communism. Notre Dame In: Notre Dame

. Le bolchevisme. Introduction historique et doctrinale. Paris: Beauchesne, 1933.

. Bolschewismus als Weltgefahr. Luzern: Vita nova, 1935.

. Le bolchevisme, danger mondial. Paris: Alsatia, 1933.

ISTRATI, Panaït. Vers l'autre flamme. Après seize mois dans l'U.R.S.S. Paris: Riëder, 1929. Réed. Paris: U.G.E., 1980.

JÜNGER, Ernst. L'État universel suivi de La mobilisation totale. Paris: Gallimard, 1990.

Kolloquium in Institut für Zeitgeschichte am 24 Nov. München: Oldenbourg, 1980.

KOSELLECK, Reinhart. L'expérience de l'histoire. Paris: Gallimard/Le Seuil, p. 101, 1997.

LINZ, Juan. Régimes totalitaires et autoritaires. Préf. de Guy Hermet. Paris: Armand Colin, p. 20, 2006.

MAIER, Hans. Das Doppelgesicht des Religiösen. Dans: Religion. Gewalt. Politik. Freiburg iB: Herder, 2004.

MISES, Ludwig V.. Omnipotent Government: The Rise of Total State and Total War. New Haven CT: Yale UP, 1944. rééd. 1969, Grove City PA: Libertarian Press.

MOORHOUSE, Roger. The Devil's Alliance. Hitler's Pact with Stalin 1939 - 1941. New York: Basic Books, 2014. 
NEUMANN, Sigmund. Permanent Revolution. The Total State in a World at War. New York, London: Harper, 1942.

ORWELL, George. 1984. Paris: Gallimard «Folio », p. 118, 1991. Trad.: A. Audiberti.

REICH, Wilhelm. La psychologie de masse du fascisme. Paris: Payot, 1972.

TORMEY, Simon. Making sense of Tyranny: Interpretations of Totalitarianism. Manchester: Manchester UP, 1995.

TRAVERSO, Enzo. Le Totalitarisme. Le 20e siècle en débat. Paris: Seuil, 2001.

TRENTIN, Silvio. Dix ans de fascisme totalitaire. Paris: Éd. sociales internationales, p. 9, 1937.

\section{Revues}

Le Figaro, Orwell, selon Simon Leys, nov., 2006.

Spartacus: No 50, Série B, Paris, oct. - nov., 1972.

The Review of Politics. Idéologie et terreur, introduction. Paris: Hermann, 2008.

Recebido em: 22/05/2019; Aceito em: 25/05/2019 